\title{
SLEEP STUDIES IN COPD PATIENTS
}

\author{
C. Yugandhar Bhatt1, Methuku Narender², Vavilala Satish Kumar Rao ${ }^{3}$, Auzumeedi Sai Kumar ${ }^{4}$, Subhakar Kandi5, Methuku Anurag ${ }^{5}$ \\ ${ }_{1}^{1}$ Assistant Professor, Department of Pulmonary Medicine, Deccan College of Medical Sciences, Hyderabad. \\ ${ }^{2}$ Associate Professor, Department of Pulmonary Medicine, Guntur Medical College, Guntur. \\ ${ }^{3}$ Assistant Professor, Department of Pulmonary Medicine, Osmania Medical College, Hyderabad. \\ ${ }^{4}$ Professor \& HOD, Department of Pulmonary Medicine, Osmania Medical College, Hyderabad. \\ 5 Professor, Department of Pulmonary Medicine, Osmania Medical College, Hyderabad. \\ ${ }^{6}$ Intermediate BiPC Student, Chaitanya Jr. Kalasala, Hyderabad.
}

\begin{abstract}
\section{BACKGROUND}

Sleep is a highly organized, complex behaviour characterized by a relative disengagement from the outer world and variable, but specific brain activity. It is an endogenously generated, homeostatically regulated and reversible. During sleep, there are profound physiological changes. This is particularly true of breathing and in number of conditions this had important implications. Breathing alters according to the state of consciousness. In healthy subjects, ventilation falls with the onset of sleep and is reduced during all phases of sleep compared with waking levels, alterations in the pattern of breathing occur including periodic breathing, apnoea, hypopnoea with gradual progression from stage I, II (18\% decreased) to REM sleep (35\% of decrease ventilation to awake stage). REM sleep related alterations in ventilation is more severe in respiratory disordered patients who had already some Hypoxemia, COPD is of best example.
\end{abstract}

\section{METHODS}

Patients who attended outpatient Department of Pulmonary Medicine, Govt. General and Chest Hospital, OMC, Hyderabad, Telangana, between May 2012 and April 2014 with clinical features s/o COPD were admitted, thoroughly examined and evaluated with routine investigations and specific investigations like sputum for AFB, Gram's stain, PFT, Chest X-ray to establish the diagnosis of COPD. (GOLD guidelines) All these patients $(n=25)$ filled the COPD questionnaire along with Sleep symptoms questionnaire and Epworth Sleep Scoring (ESS). These patients are advised to undergo whole night PSG after informed consent.

\section{RESULTS}

The 24 patients with COPD were divided into two groups, Group 1: 6 patients out of 24 (25\%) showed significant OSAHS (AHI $>5 / \mathrm{hr}$., i.e. overlap syndrome); Group 2: Rest of the 18 patients (75\%) showed no significant OSAHS. Among these 18 patients, 12 are having asymptomatic hypopneas (Clinically insignificant SDB), 6 are not significant OSAHS. Among these 2 groups, mean values of 25 variables were tabulated, compared and analysed by using window stat version 8.5 from IndoStat services. Among the 25 variables, 11 were significantly different between 2 groups.

\section{CONCLUSIONS}

There is positive correlation between neck circumference, BMI and Epworth score with AHI (Apnoea-Hypopnea Index). There is positive correlation between frequency of arousals, low sleep efficiency, REM sleep with $\mathrm{O}_{2}$ drop and snoring associated arousals with AHI. There is negative correlation between severity of COPD, duration of COPD and smoking with AHI. OSAHS in COPD patients (Overlap Syndrome) is incidental and does not share the pathology of two diseases (COPD and OSA). Sleep changes like reduced REM sleep, largest oxygen saturation drop during REM sleep, frequent arousals, frequent changing of sleep stages are observed in COPD patients, but more significantly seen in overlap syndrome. Independent risk factors for OSAHS like high BMI, more neck circumference, facial abnormalities and symptoms like excessive day time sleepiness should be looked for diagnosing overlap syndrome in COPD patients.

\section{KEYWORDS}

OLS (Overlap Syndrome); OSAHS (Obstructive Sleep Apnoea Hypopnoea Syndrome); PSG (Polysomnography); AHI (Apnoea Hypopnoea Index); COPD (Chronic Obstructive Pulmonary Disease); CPAP (Continuous Positive Airway Pressure).

HOW TO CITE THIS ARTICLE: Bhatt CY, Narender M, Rao VSK, et al. Sleep studies in COPD patients. J. Evolution Med. Dent. Sci. 2016;5(32):1759-1762, DOI: 10.14260/jemds/2016/414

\section{INTRODUCTION}

Sleep is a highly organized, complex behaviour characterized by a relative disengagement from the outer world and

Financial or Other, Competing Interest: None.

Submission 20-03-2016, Peer Review 02-04-2016,

Acceptance 04-04-2016, Published 21-04-2016.

Corresponding Author:

Dr. C. Yugandhar Bhatt

Assistant Professor,

Department of Pulmonary Medicine,

Deccan College of Medical Sciences,

Hyderabad.

E-mail:drugandhar@gmail.com

DOI: $10.14260 /$ jemds/2016/414 variable, but specific brain activity. It is an endogenously generated, homeostatically regulated and reversible. During sleep, there are profound physiological changes. This is particularly true of breathing and in number of conditions this had important implications.

Breathing alters according to the state of consciousness in healthy subjects, ventilation falls with the onset of sleep and is reduced during all phases of sleep compared with waking levels.(1)

Alterations in the pattern of breathing occur including periodic breathing Apnoea, Hypopnea with gradual progression from Stage I, II (18\% decreased) to REM sleep 
(35\% of decrease ventilation to awake stage).

REM sleep related alterations in ventilation is more severe in respiratory disordered patients, who had already some Hypoxemia, COPD is of best example.

\section{As patients with COPD have}

I) A higher physiological dead space than normal subjects and therefore the rapid shallow breathing that occurred during sleep will produce an even greater decrease in Alveolar ventilation.(2)

II) Hyperinflation of lungs in COPD patients leads to more dependency upon Intercostal and Accessory muscles activity than normal individuals, but during REM sleep these Intercostal and Accessory Muscles are Flaccid, hence Diaphragm alone is unable to meet the demand.

III) Decrease FRC, V/Q Mismatch during sleep in COPD patients. $(3,4,5)$ OSAHS is SRBD of which shows oxygen desaturation during REM sleep.

If both OSAHS and COPD coexist in an individual, REM sleep related oxygen desaturation is more severe and as a result leads to many Cardiac, Non-Cardiac complications like Fluid Retention.(6) polycythaemia.(7) Cardiac arrhythmia.(8) Pulmonary Hypertension.(9) Hence, their early diagnosis and management is necessary.

All above changes should be well identified by doing Polysomnography (PSG) in COPD patients, where we know the (a) Impact of sleep on COPD, (b) Influence of COPD on sleep pattern. ${ }^{(10,11)}$

Several studies demonstrate $\mathrm{PaO}_{2}$ decreased during sleep in COPD patients. ${ }^{(12,13)}$ mainly during REM sleep.(14,15), being this decrease more pronounced during the Tonic phase of this sleep stage.(16) It is natural to believe that Hypoxemia is responsible for sleep fragmentation in COPD and this Hypothesis is supported by experimental studies.(2) and in diffuse parenchymal pulmonary disease patients. $(17,18)$

Another Hypothesis to explain sleep fragmentation is the Hypercapnia, demonstrated in some studies.(19,20) subjects wake up with $\mathrm{PaCO}_{2}$ close to $55 \mathrm{mmHg}$ during NREM sleep and $60 \mathrm{mmHg}$ during REM sleep. Paradoxically predominantly Emphysema patients, usually Non-Hypercapnic and NonHypoxemic are the ones who present more sleep fragmentation suggesting that yet another factor such as increased ventilatory effort is responsible for awakening.(21) In addition, more Hypoxemic individually present a better sleep pattern, although they can evolve to Cardiac arrhythmia.(22) Right Ventricular Hypertrophy.(23) Polycythaemia.(24) and Pulmonary Hypertension.(25), the correlation between ventilatory functional test of COPD patients and PSG variables are still little studied.

This study as the objective of examining possible correlations among spirometric and polysomnographic variables in COPD patients.

\section{AIMS AND OBJECTIVES}

There is paucity of literature regarding sleep in COPD patients, particularly use of sleep studies in these patients. No direct study is seen in Indian population. As studies already done were giving variable prevalence and conclusions, we have conducted this study with the objective of,

a) Impact of sleep in COPD patients.

b) Influence of COPD on sleep pattern (Quality of sleep).

c) Overlap syndrome-prevalence.

\section{Study Design}

This is a prospective, analytical, transversal type of study.

\section{MATERIAL AND METHODS}

Patients who attended Outpatient Department of Pulmonary Medicine, Telangana Govt. General and Chest Hospital, OMC, Hyderabad, between May 2012 and April 2014 with clinical features s/o COPD were admitted, thoroughly examined and evaluated with routine investigations and specific investigations like sputum for AFB, Gram's stain, PFT, Chest Xray to establish the diagnosis of COPD. (GOLD guidelines).

All these patients $(n=25)$, filled the COPD questionnaire along with Sleep Symptoms Questionnaire and Epworth Sleep Scoring (ESS).

These patients are advised to undergo whole night PSG after informed consent.

This study was considered as minimum risk in human research and was approved by the Ethics Committee of the University.

\section{PROCEDURE}

\section{Inclusion Criterion}

1. Patients having SOB, cough, history of smoking.

2. $\mathrm{FEV}_{1} / \mathrm{FVC}<0.7$ with irreversibility.

3. Snoring +ve and nocturnal sleep disturbance with or without EDS.

\section{Exclusion Criterion}

1. Asthma.

2. Other respiratory diseases.

3. Cor-pulmonale.

4. Patients who slept less than 4 hours.

Among these 25 selected patients of COPD with various degree of severity, one patient was excluded as he slept less than 4 hours.

These 24 patients underwent anthropometry in which weight and height were measured to the nearest 500 gms and $1 \mathrm{~cm}$ respectively and the BMI was calculated based on the formula. BMI= weight $(\mathrm{kgs}) /$ height ${ }^{2}\left(\mathrm{cms}^{2}\right)$.

Neck circumference (cms) was measured at the level of cricothyroid membrane. Patients have reported to the sleep laboratory of the Department of Pulmonary Medicine at 8 PM on the day of testing.

All these patients underwent whole night Polysomnography. PSG is done by 54 multi-channel ALICE- 5 system with whole night video recording. The entire testing process was done under the supervision of trained and capable technicians following the specifications and the criterion established by the $\mathrm{R}$ and $\mathrm{K}$ system.

\section{In PSG the following Montages were used}

1. EEG-7 sites-for staging of sleep (Gold coated cup electrodes are used).

2. EOG-1 cm upwards and outwards from the right outer canthus.

$1 \mathrm{~cm}$ downwards and outwards from the left outer canthus.

3. CEMG-2 sites-one above and one below to the chin for muscle tone measurement and reorganization of Bruxism.

4. LEMG-4 sites-two on each side-over shin and lower tibiafor the measurement of muscle tone and identification of PLMI. 
5. Abdominal and thoracic belts-for identification of efforts.

6. Nasal Cannula - For the measurement of air flow.

7. Positional sensor - For posture of patient.

8. Pulsoximeter - for $\mathrm{SpO}_{2}$ measurement.

9. EKG - For cardiac monitoring.

10. Video monitoring.

Patient was requested to sleep around $10 \mathrm{PM}$. The recording of sleep study was started after ensuring the impedance of the electrodes was set to zero. Raw data is analysed manually. Sleep stage is scores as per $\mathrm{R}$ and $\mathrm{K}$ rules. Respiratory events are scored according to revised AASM task force recommendations.

\section{RESULTS}

The 24 patients with COPD were divided into two groups Group 1: 6 patients out of 24 (25\%) showed significant OSAHS (AHI $>5 /$ hr., i.e. overlap syndrome); Group 2: Rest of the 18 patients (75\%) showed no significant OSAHS. Among these 18 patients, 12 are having asymptomatic hypopneas (Clinically insignificant SDB), 6 are not significant OSAHS. Among these 2 groups, mean values of 25 variables were tabulated, compared and analysed by using Window Stat Version 8.5 from IndoStat services.

Among the 25 variables, 11 were significantly different between 2 groups.

1) Epworth score, which is indicative of excessive daytime sleepiness, well correlating with AHI, i.e. high $(>10)$ in Group 1, less in Group 2.

2) Neck circumference and body mass index were high among Group 1, low in Group 2 patients according to student $T$ test $(p=0.009$ and $p=0.002)$ and Mann Whitney $(p=0.002$ and $p=0.001)$

It is suggesting that clinical features (eg: EDS and smoking) and anthropometric findings (eg: NC and BMI) are useful to screen OSAHS.

3) Duration of NREM sleep is low in Group 1 patients according to $\mathrm{T}$ test $(\mathrm{p}=0.001)$ and Mann Whitney $(\mathrm{p}=0.000)$ and TAI more in Group 1 according to T test $(\mathrm{p}=0.014)$ and Mann Whitney $(\mathrm{p}=0.000)$.

4) TAI (Total Arousal Index) with oxygen drop/AHI (Apnoea Hypopnoea Index) and OA are high in Group 1 patients.

According to $\mathrm{T}$ test $(\mathrm{p}=0.000)$ and Mann Whitney $(p=0.000)$, the inference is that obstructive apnoea is causing more number of arousals with significant oxygen drop among this group, which results in complications earlier than Group 2.

5) Desaturation with NREM sleep: TAI is more pronounced in Group 1 patients according to $\mathrm{T}$ test $(\mathrm{P}=0.005$ and 0.014 ) and Mann Whitney ( $\mathrm{p}=0.0052$ and 0.020$)$.

\section{DISCUSSION}

Though there is no common pathological link between OSAHS and COPD, their coexistence is not uncommon. The patients with clinical features suggesting OSAHS should be evaluated with Epworth score and overnight PSG for early detection of OSAHS.

Hence, sleep study in COPD patients even though not indicated in all COPD patients but should be done at least in high risk patients to avoid premature cardiac and non-cardiac complications secondary to OSAHS.

\section{Limitations of Study}

Arterial blood gas analysis not done, oesophageal pressure monitoring is not done. Sample size too small to know the prevalence of overlap syndrome among COPD patients.

\begin{tabular}{|c|c|c|c|c|}
\hline GOLD/AHI & $\mathbf{0 - 5}$ & $\mathbf{5 - 1 5}$ & $\mathbf{1 5 - 3 0}$ & $>\mathbf{3 0}$ \\
\hline Category 1 & 0 & 0 & 0 & 0 \\
\hline Category 2 & 0 & 2 & 2 & 0 \\
\hline Category 3 & 2 & 3 & 1 & 1 \\
\hline Category 4 & 4 & 4 & 2 & 3 \\
\hline \multicolumn{5}{|c|}{ Table 1 } \\
\hline
\end{tabular}

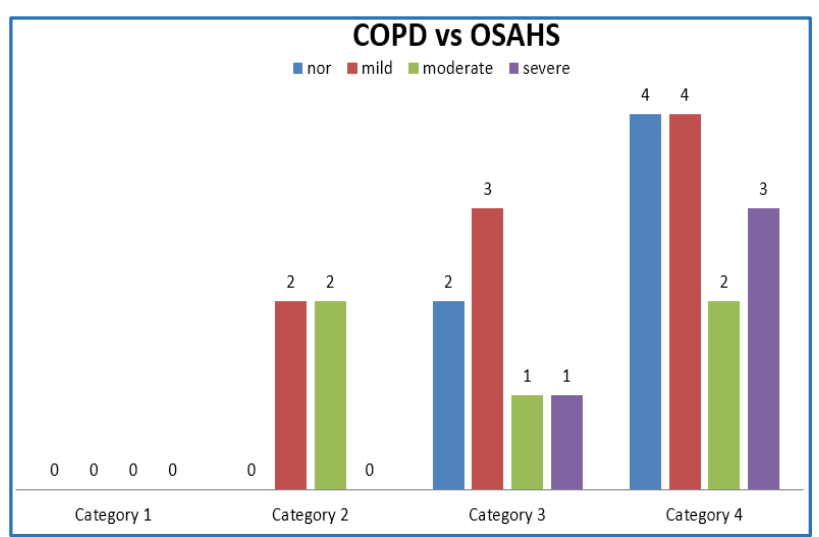

Fig. 1

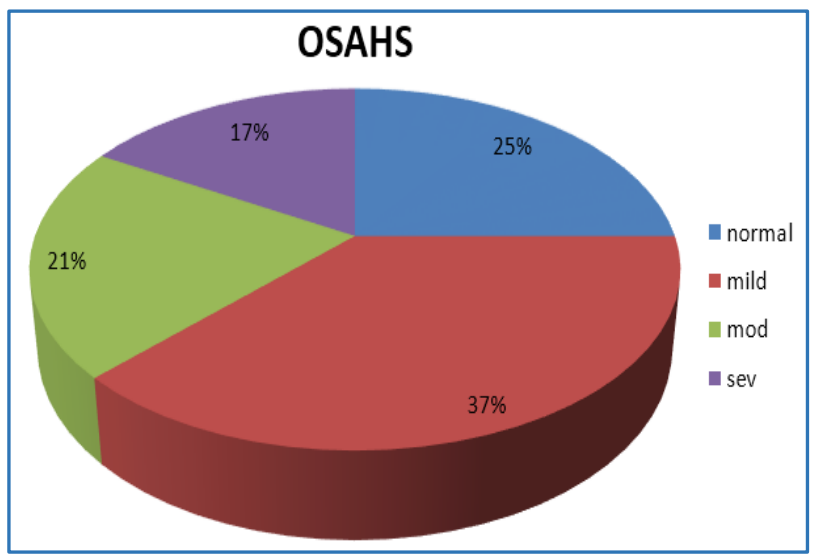

Fig. 2

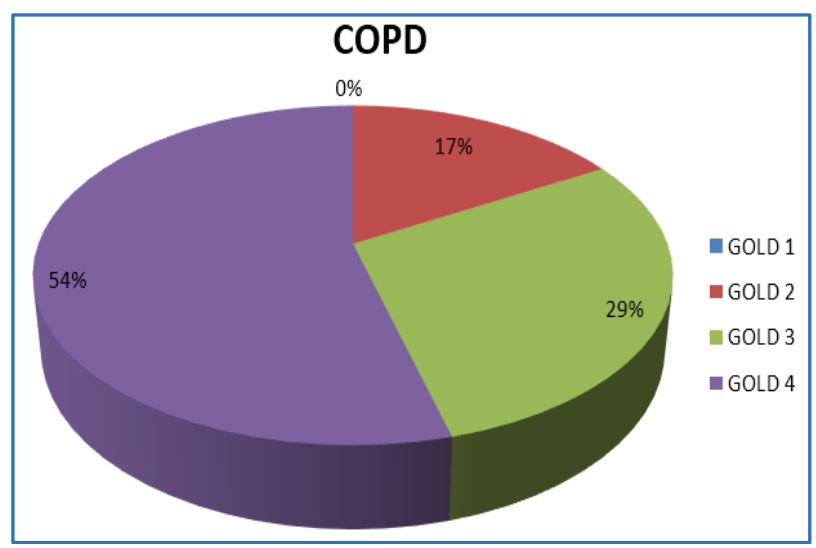

Fig. 3 


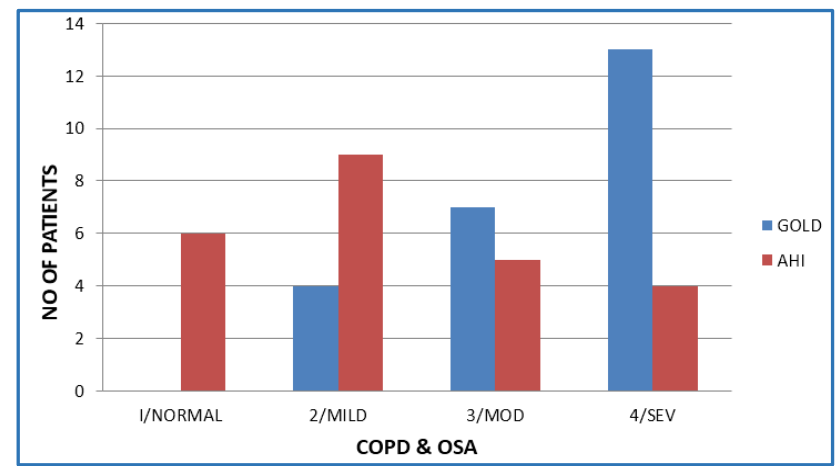

Fig. 4

\section{CONCLUSIONS}

There is positive correlation between neck circumference, BMI and Epworth score with AHI (Apnoea-Hypopnoea Index).

There is positive correlation between frequency of arousals, low sleep efficiency, REM sleep with 02 drop and snoring associated arousals with AHI.

There is negative correlation between severity of COPD, duration of COPD and smoking with AHI.

OSAHS in COPD patients (Overlap Syndrome) is incidental and does not share the pathology of two diseases (COPD and OSA).

Sleep changes like reduced REM sleep, largest oxygen saturation drop during REM sleep, frequent arousals, frequent changing of sleep stages are observed in COPD patients, but more significantly seen in overlap syndrome.

Independent risk factors for OSAHS like high BMI, more neck circumference, facial abnormalities and symptoms like excessive day time sleepiness should be looked for diagnosing overlap syndrome in COPD patients.

\section{Clinical Implication}

Though there is no common pathological link between OSAHS and COPD, their core existence will not be uncommon. With various clinical features, these suspicious clinical features should be evaluated with Epworth score and thorough investigation with overnight PSG for early detection of OSAHS.

Hence, sleep studies in COPD patients even though not indicated in all COPD patients, but should be done at least in high risk group patients to avoid cardiac and non-cardiac premature complications.

\section{REFERENCES}

1. Douglas NJ, White DP, Pickett CK, et al. Respiration during sleep in normal man. Thorax 1982;37(11):840-4.

2. Moore-Gillon JC, Cameron IR. Right ventricular hypertrophy and polycythemia in rats after intermittent exposure to hypoxia. Clin Sci 1985;69(5):595-9.

3. Cameron D Harris. Neurophysiology of sleep and wakefulness. Respiratory care clinics of North America 2005;11(4):567-86.

4. Samuel Krachman, Omar A Minai, Steven M Scharf. Sleep abnormalities and treatment in emphysema. Proc Am Thorac Soc 2008;5(4):536-42.

5. Hobson JA, McCarley RW, Wyzinski PW. Sleep cycle oscillation: reciprocal discharge by two brainstem neuronal groups. Science 1975;189(4196):55-8.

6. Littner NR, McGnity DJ, Arand DL. Determinants of 02 desaturation in the cause of ventilation during sleep in COPD. Am Rev Respir Dis 1980;122:849-57.
7. Celli BR, MacNee W. Standards for the diagnosis and treatment of patients with COPD: a summary of the ATS/ERS position paper. Eur Respir J 2004;23(6):932-46.

8. Orr WC, Sharmma-Othman Z, Levin D, et al. Persistant hypoxemia and EDS in COPD. Chest 1990;97(3):583-5.

9. De Miguel J, Cabello J, Sanchez-Alarcos JM, et al. Longterm effects of treatment with nasal CPAP on lung function in patients with overlap syndrome. sleep breath 2002;6(1):3-10.

10. Meoli AL, Casey KR, Clark RW, et al. Hypopnea in sleep disordered breathing in adults. Sleep 2001;24(4):46970.

11. Fletcher EC, Gray BA, Levin DC. Non apneic mechanism of arterial 02 desaturation during REM sleep. J Appl physio 1983;54:632-9.

12. Guilleminault $\mathrm{C}$, Stoohs R, Clerk A, et al. A cause of excessive daytime sleepiness. The UARS. Chest 1993;104(3):781-7.

13. Chaouat A, Wetzenbum E, Krieger J, et al. Association of COPD and sleep apnea syndrome. AM J Respir Crit Care Med 1995;151(1):82-6.

14. Douglas NJ, Flenley DC. Breathing during sleep in COPD patients. Am Rev Respir Dis 1990;141:1055-70.

15. Koo KW, Sax DS, Snider GL. ABG tensions and $\mathrm{pH}$ during sleep in COPD. Am j med 1975;58(5):663-70.

16. Leitch AG, Clancy LJ, Leggett RJE, et al. Arterial blood gas tensions, hydrogen ion \& EEG during sleep in patients with chronic ventilatory failure. Thorax 1976;31:730-5.

17. Fitzpatrick MF, Mackay T, Whyte KF, et al. Nocturnal desaturation and serum erythropoietin: a study in patients with chronic obstructive pulmonary disease and in normal subjects. Clin Sci 1993;84(3):319-24.

18. Shepard JW, Garrison MW, Grither DA, et al. Relationship of ventricular ectopy to nocturnal oxygen desaturation in patients with chronic obstructive pulmonary disease. Am J Med 1985;78(1):28-34.

19. Boysen PG, Block AJ, Wynne JW, et al. Nocturnal pulmonary hypertension in patients with chronic obstructive pulmonary disease. Chest 1979;76(5):53642.

20. John A Fleetham. Is chronic obstructive pulmonary disease related to sleep apneahyponea syndrome? American journal of Respiratory and critical care medicine 2003;167(1):3-4.

21. Catteral JR, Calverly PM, MacNee W, et al. Mechanism of transient nocturnal hypoxemia in hypoxic COPD. J Appl Physiology 1985;59(6):1698-703.

22. Goldstein RS, Ramcharan V, Bower G, et al. Effect of supplemental nocturnal oxygen on gas exchange in pts with severe obstructive lung disease. NEJM 1984;310(7):425-9.

23. Emmanuel Weitzenblum, Ari Chaouat, Romain Kessler, et al. Department de pneumologie, ho pitaux universitaries de strasbourg, strasbourd, France; and 2 service des maladies respiratories et re. Animation respiratoire, centre hospitalier universitaires de nancy, vandoeuvre les nancy, France proc. Am Thorac soc 2008;5:237-41.

24. Douglas NJ, Calverley PM, Leggett RJ, et al. Transient hypoxemia during sleep in chronic bronchitis \& emphysema. Lancet 1979;1(8106):1-4.

25. Bengt Midgren, Lennart Hansson, Havard Skeidsvoll, et al. Nocturnal hypoxemia in COPD. Chest 1989;95(4):7658. 